\title{
The effect of increased fruit and vegetable intake on diet profile in a diabetic population: Fruit and Vegetables in Diabetes (FVD) study
}

\author{
S. L. Fulton ${ }^{1}$, C. McLaughlin ${ }^{2}$, D. R. McCance ${ }^{2}$, M. C. McKinley ${ }^{1}$, I. S. Young ${ }^{1}$ and J. V. Woodside ${ }^{1}$ \\ ${ }^{1}$ Nutrition and Metabolism Group, Centre for Public Health, Queen's University Belfast, Grosvenor Road, Belfast, \\ UK, BT12 6BJ and ${ }^{2}$ Regional Centre for Endocrinology, Belfast Health and Social Care Trust, Grosvenor Road, \\ Belfast, BT12 6BA, UK
}

Research predominantly focuses on high levels of antioxidants, flavonoids and polyphenols contained in fruit and vegetables (FVs) as the beneficial components to health; however, increasing FV may also alter overall diet profile. Furthermore FV variety may have an effect on disease risk ${ }^{(1)}$, yet it is not known whether increasing FV quantity also necessarily leads to an increase in variety. Therefore the aims of this research were, firstly to determine the effect of increasing FV intake on overall diet profile and, secondly, to examine how participants asked to increase FV intake achieved this.

Data was collected as part of an 8-week randomised controlled trial of diabetic patients, where FV intake increased to $\geq 6$ portions/ day or participants followed a low FV diet ( $\leq 1$ portion/day), after a 4 week run-in period. Subjects collected 7-day food diaries, which were analysed using WISP-DESv3.0. Daily portion counts were manually counted using household measures established by the Department of Health ${ }^{(2)}$.

Between group analysis of changes in macronutrient intakes (see table below), revealed significant differences in the majority of nutrients, but not energy intake, when FV intake increased. Between group analysis of changes in micronutrient intakes revealed significant differences in intakes of vitamins $\mathrm{C}$ and $\mathrm{E}$, carotene, magnesium, potassium, and folate. The lack of difference in change in energy intake between the groups suggests there was substitution of FVs for other potentially less healthy foods.

\begin{tabular}{|c|c|c|c|c|c|c|c|c|c|c|c|c|}
\hline & \multicolumn{6}{|c|}{ 1/day $\left(n_{\max }=38\right)$} & \multicolumn{6}{|c|}{ 6/day $\left(n_{\max }=34\right)$} \\
\hline & \multicolumn{2}{|c|}{ Wk 0} & \multicolumn{2}{|c|}{ Wk 8} & \multicolumn{2}{|c|}{ Change } & \multicolumn{2}{|c|}{ Wk 0} & \multicolumn{2}{|c|}{ Wk 8} & \multicolumn{2}{|c|}{ Change } \\
\hline & Mean & SD & Mean & SD & Mean & $95 \% \mathrm{CI}$ & Mean & SD & Mean & SD & Mean & $95 \% \mathrm{CI}$ \\
\hline Energy (kcals/d) & 1601 & 370 & 1646 & 424 & 59.8 & $-74.1,194$ & 1652 & 494 & 1748 & 486 & 109 & $-65.4,284$ \\
\hline Total fat (g/d) & 40.7 & 6.81 & 40.0 & 5.41 & -0.76 & $-2.83,1.31$ & 41.2 & 5.97 & 36.8 & 6.36 & -3.74 & $-6.13,-1.34$ \\
\hline SFA $(\mathbf{g} / \mathbf{d})$ & 15.5 & 3.84 & 15.5 & 3.84 & 0.18 & $-0.79,1.15$ & 15.3 & 3.36 & 13.2 & 3.00 & $-1.70 *$ & $-2.88,-0.51$ \\
\hline MUFA (g/d) & 14.3 & 3.01 & 14.3 & 2.39 & 0.07 & $-0.80,0.95$ & 13.8 & 2.28 & 12.2 & 1.82 & $-1.40 *$ & $-2.35,-0.44$ \\
\hline $\operatorname{Carbs}(\mathrm{g} / \mathrm{d})$ & 113 & 18.4 & 113 & 15.1 & -0.74 & $-5.14,3.65$ & 111 & 14.8 & 123 & 14.3 & $10.3 * *$ & $4.53,16.1$ \\
\hline Starch $(g / d)$ & 75.8 & 14.1 & 75.1 & 13.1 & -1.11 & $-4.77,2.55$ & 75.4 & 12.7 & 64.7 & 11.6 & $-11.8 * *$ & $-16.9,-6.67$ \\
\hline Total sugar $(g / d)$ & 36.1 & 17.5 & 37.1 & 13.4 & 0.45 & $-3.67,4.57$ & 34.7 & 10.2 & 56.6 & 14.0 & $21.7 * * *$ & 16.0, 27.4 \\
\hline Dietary fibre (g/d) & 7.49 & 2.35 & 7.41 & 2.06 & -0.28 & $-0.93,0.36$ & 7.38 & 2.45 & 10.3 & 2.35 & $2.93 * * *$ & $1.81,4.04$ \\
\hline
\end{tabular}

$* p<0.05 ; * * p<0.01 ; * * * p<0.001$. Change calculated as wk $8-$ wk 0 . Independent samples t-test.

Peas, baked beans and carrots were the most common vegetable choices, whilst bananas, apples and tomatoes were the most common fruit choices. The number of different FV choices made at week 8, in the 1 and 6 portion groups respectively, were fruit: 24 , 35, and vegetables: 26,44 . Therefore, individuals chose a greater variety of FVs as opposed to just larger quantities of the same FV when asked to increase intakes. Breakfast was the most popular time for fruit intake, and lunch and dinner for vegetable intake.

In conclusion, when diabetic participants increased FV intake, an improvement was observed in overall diet profile, with an increase in the variety of FV consumed, no significant difference in energy, decreases in saturated and monounsaturated fats and increases in carbohydrates, total sugars, dietary fibre and a number of micronutrients.

1. Bhupathiraju SN \& Tucker KL (2011) Am J Clin Nutr 93, 37-46.

2. NHS (2009). http://www.nhs.uk/LiveWell/5ADAY/Pages/5ADAYhome.aspx (accessed June 2011). 